\title{
Gain Adaptation Policies for Dual-Hop Nonregenerative Relayed Systems
}

\author{
Marios A. Gatzianas, Leonidas G. Georgiadis, Senior Member, IEEE, \\ and George K. Karagiannidis, Senior Member, IEEE
}

\begin{abstract}
We examine the performance of a dual-hop nonregenerative system with adjustable relay gain, subject to power constraints. An optimization problem is formulated and solved algorithmically for the binary phase-shift keying bit-error rate utility. The model allows for arbitrary channel statistics. Emphasis is placed on the relation between the optimal solutions obtained when observing the channels of either the first or both hops, as well as the comparison with easily implementable heuristic policies. $\mathrm{Nu}$ merical results indicate that simple heuristics perform well for a wide range of signal-to-noise ratio (SNR), except for certain highSNR cases. Finally, the effect of independent channel assumption on system performance is evaluated.
\end{abstract}

Index Terms-Convex optimization, dual-hop systems, fading channels.

\section{INTRODUCTION}

$\mathbf{R}$ ELAYED transmission is a promising technique for improving the quality of wireless communications. Its advantages relative to direct link communication include, among others: ease of implementation and good scalability, increased connectivity, robustness to changing channel conditions, and reduced operating power levels. The latter implies lower interference levels and, hence, increased capacity. In further support of the above advantages, comes the concept of cooperative user diversity, which can potentially offer even higher capacity with a reduced outage probability [1]-[4].

Since cooperative diversity depends on relays to achieve its goals, the two techniques have been traditionally examined together, and a certain terminology has evolved [5] (regenerative versus nonregenerative, blind/semi-blind/channel-stateinformation (CSI), etc.).In [5], the authors examine a semiblind relay and compare a regenerative to a nonregenerative system, while [6] presents an instantaneous-power-constrained optimization problem for a dual-hop scenario and offers a generalization to a multihop regenerative system. In both cases, Rayleigh fading is assumed, and the outage probability is used as the performance metric (see [7] for a more general setting).

Boyer et al. examine in [8], for an isolated single-user link, various combinations of the above cases in a "serial" multihop scenario, where each relay can potentially receive all signals transmitted by the previous relays, while [9] proposes distributed codes for a "parallel" multihop case where the original transmitter broadcasts to all relays, and a suitably selected subset of them

Paper approved by Y. Fang, the Editor for Wireless Communication of the IEEE Communications Society. Manuscript received July 3, 2005; revised April 7, 2006 and accepted October 25, 2006. This work was presented in part at the IEEE International Conference on Communications, Istanbul, June 2006.

The authors are with the Department of Electrical and Computer Engineering, Division of Telecommunications, Aristotle University of Thessaloniki, Thessaloniki 54124, Greece (e-mail: mgkatzia@auth.gr; leonid@auth.gr; geokarag@auth.gr).

Digital Object Identifier 10.1109/TCOMM.2007.902530 retransmits to the desired destination. In a similar context, [10] examines the effect of relay cooperation on the overall capacity region. Since diversity is outside the scope of this paper, it will not be further discussed although it will become apparent that the ultimate goal is to incorporate it into our proposed model.

In this work, we consider a dual-hop nonregenerative system with arbitrary fading statistics. The relay node may have knowledge of the CSI of either the first only or of both channelstransmitter to relay and relay to receiver. Our objective is to investigate the effect of the relay's knowledge of each channel's fading state on the overall performance. In addition to instantaneous power constraints (such as those appearing in [6]), we also impose a constraint on either the (long term) average power output by the relay or the average power consumed by it, the latter defined as the difference between the power output by the relay and the power received by it. ${ }^{1}$ The consumption constraint is especially useful in cases where the relay is mobile and relies on batteries (hence, limited energy) for its operation.

System performance is formulated as a constrained nonlinear optimization problem with concave performance objective, which may include average signal-to-noise ratio (SNR), biterror rate (BER), or Shannon rate. The control to be exercised is the relay retransmission power when some of the channel states are observed. We present the methods for computing this control depending on the available channel states, and examine the benefits of observing either the first channel or both. Additionally, using the derived optimal solution as a benchmark, we evaluate the performance of suboptimal policies such as a modified inverse gain formula (in the spirit of [11]) and pure SNR-based optimization. The comparison results are interesting and, to a certain extent, unexpected.

\section{SySTEM AND CHANNEL MODEL}

Consider a transmitter, relay, and receiver that communicate over wireless channels. Denote the channels between transmitter-relay and relay-receiver as channels 1 and 2, both exhibiting random fading with coefficients $a$ and $b$, respectively, defined as the appropriate power ratios between the transmitter and receiver for each channel. We denote as $\mathcal{A}, \mathcal{B}$ the finite sets over which $a$ and $b$ range, respectively. This assumption, which seems to contradict the continuous nature of fading, is imposed in order to reduce the mathematical complexity of the problem. Though a treatment of continuous fading sets is provided in [12] using calculus of variations, the main concepts are essentially the same. Specifically, since all constraints are either pointwise or appear inside a summation, it can be shown that convergence to the continuous solution is achieved as $|\mathcal{A}|,|\mathcal{B}| \rightarrow \infty$.

\footnotetext{
${ }^{1}$ This distinction creates two problem variants, hereafter, referred to as the "output" and "consumption" variant, respectively.
} 
In each channel, a positive power gain $\left(g_{1}\right.$ and $g_{2}$, respectively) is used to amplify the base signal at the corresponding transmitter. Hence, assuming that the first transmitter transmits at unity power and the relay and receiver are subject to additive white Gaussian noise (AWGN) of powers $N_{1}$ and $N_{2}$, respectively, it is easy to show that the instantaneous SNR at the receiver (end-to-end), conditioned upon the fading coefficients $a, b$, is given by

$$
\gamma_{a, b}=\frac{g_{1} g_{2} a b}{N_{2}+b g_{2} N_{1}}=\frac{g_{1} a}{N_{1}} \frac{b g_{2}}{b g_{2}+N_{2} / N_{1}} .
$$

We, hereafter, denote $\eta=N_{2} / N_{1}$. The term "instantaneous" is used here in a loose context to denote a time average of the randomly varying SNR over a time interval equal to the channel's coherence time. Since, by definition, $a$ and $b$ remain constant in such an interval, $\gamma_{a, b}$ is actually a short-term time average, similar in concept to [13], and it is in this context that we can regard it as instantaneous. The long-term SNR time average is by ergodicity equal to the statistical average over all fading values.

The transmitter gain $g_{1}$ is arbitrarily fixed, whereas $g_{2}$ is allowed to vary as a (yet unknown) deterministic function of $a, b$, or only $a$. The various dependencies physically correspond to different fading states being known at the relay, i.e., the relay bases its decisions on the knowledge of both $a, b$, or only $a$. The case where the relay observes $b$ only is of little practical interest and has received little, if any, attention since the relay can always estimate $a$ from its incoming signal $g_{1} a+N_{1}$ (assuming that $g_{1}$ and $N_{1}$ are known). Therefore, the $b$ only dependence will not be examined in this paper.

\section{Problem Statement}

Since $\gamma_{a, b}$ is a random variable, we take the expectation of a utility function of it as a figure of merit, and seek to maximize this subject to average and instantaneous power constraints as

$$
\begin{aligned}
& \underset{g_{2}}{\operatorname{maximize}} \mathbb{E}\left\{\Phi\left(\gamma_{a, b}\right)\right\} \\
& \text { s.t. } 0 \leq g_{2}\left(g_{1} a+N_{1}\right) \leq p_{2} \\
& \quad 0 \leq \mathbb{E}\left\{g_{2}\left(g_{1} a+N_{1}\right)\right\} \leq \bar{P}_{2} .
\end{aligned}
$$

The function $\Phi$ is required to be positive, increasing, concave, and continuously differentiable. The functions that satisfy the above properties include $\Phi(x)=x$ (i.e., maximize SNR), $\Phi(x)=\log (1+x)$ (maximize Shannon rate), and most complementary BERs (i.e., $1-P_{e}$ ) of practical modulation schemes.

Casting (2) into standard "minimize" notation results in

$$
\begin{gathered}
\underset{g_{2}}{\operatorname{minimize}} \sum_{a \in \mathcal{A}} \sum_{b \in \mathcal{B}}-\Phi\left(\frac{g_{1} a}{N_{1}} \frac{b g_{2}}{b g_{2}+N_{2} / N_{1}}\right) \pi_{a b} \\
\text { (P) } \quad \text {.t. } \quad 0 \leq g_{2}\left(g_{1} a+N_{1}\right) \leq p_{2} \\
\quad \sum_{a \in \mathcal{A}} \sum_{b \in \mathcal{B}} g_{2}\left(g_{1} a+N_{1}\right) \pi_{a b} \leq \bar{P}_{2}
\end{gathered}
$$

where $\pi_{a b}$ is the joint channel pdf and $g_{2}$ is a function of either $a$ or both $a, b$ according to the rationale of Section II. Existence of a solution to $(\mathrm{P})$ follows from continuity of $\Phi$ over the compact constraint set, while uniqueness follows from strict convexity of the objective functional.

Eqs. (3a) and (3b) describe the output variant. The consumption variant is formulated by keeping ( $3 a$ ) and ( $3 b)$ and replacing (3c) by

$$
\sum_{a \in \mathcal{A}} \sum_{b \in \mathcal{B}}\left[g_{2}-1\right]^{+}\left(g_{1} a+N_{1}\right) \pi_{a, b} \leq \hat{P}_{2}
$$

where the $[x]^{+}=\max (x, 0)$ operator is necessary to ensure nonnegative power consumption. Equation (4) is derived from the definition of instantaneous consumed power as

$$
P_{\text {cons }}=\left[P_{\text {out }}-P_{\text {in }}\right]^{+}=\left[g_{2}\left(g_{1} a+N_{1}\right)-\left(g_{1} a+N_{1}\right)\right]^{+}
$$

from which the average constraint follows trivially. The physical interpretation of the []$^{+}$operator is that when $g_{2} \leq 1$, the relay need not perform any amplification and can just let the transmitted signal pass through (hence consuming negligible power).

Finally, when there is no average power constraint (either output or consumption), the optimization problem always has the trivial solution $g_{2}=p_{2} /\left(g_{1} a+N_{1}\right)$, since the function $k x /(1+k x)$ is increasing with respect to $x, \Phi$ is increasing with respect to its argument, and the objective is a positive weighted sum of increasing functions. Hence, the standard inverse fading gain formula proposed in [11] is a special case of our general formulation.

\section{DuAl FoRMUlation: THEORY AND ALgORITHMS}

We consider the following archetype problem, special cases of which constitute the discrete problems examined in this paper:

$$
\begin{array}{ll}
\operatorname{minimize} & \sum_{j} f_{j}\left(x_{j}\right) \\
\text { s.t. } & 0 \leq x_{j} \leq p_{j}, \quad \forall j \\
& \sum_{j} c_{j} x_{j} \leq \bar{P}, \quad c_{j} \geq 0
\end{array}
$$

where $j$ ranges from 1 to $N$, and each $f_{j}$ is a continuously differentiable decreasing and strictly convex function with respect to $x_{j}$. The fact that the output variant, (3a) and (3b), falls under this formulation is obvious. The consumption variant can also be reduced to this form after some manipulations, as shown in [12].

A necessary and sufficient condition based on the duality theory for a vector $x^{*}$ to be the solution to $(6 a)-(6 c)$ is provided in [14], and since the analysis is quite standard, we only present the final results. Specifically, if $\sum_{j} c_{j} p_{j} \leq \bar{P}$, the optimal solution is $x_{j}^{*}=p_{j}, \forall j$. Otherwise, it is given parametrically by ${ }^{2}$

$$
x_{j}^{*}\left(\mu^{*}\right)= \begin{cases}G_{j}^{-1}\left(\mu^{*}\right), & \text { if } G_{j}\left(p_{j}\right) \leq \mu^{*} \leq G_{j}(0) \\ 0, & \text { if } \mu^{*} \geq G_{j}(0) \\ p_{j}, & \text { if } \mu^{*} \leq G_{j}\left(p_{j}\right)\end{cases}
$$

\footnotetext{
${ }^{2}$ For the consumption variant, 0 must be replaced by 1 in all branches of (7) and all subsequent references and the summation in (8) must be performed over the set $\left\{j: p_{j} \geq 1\right\}$.
} 
where $G_{j}(x)=-f_{j}^{\prime}(x) / c_{j}$ and the parameter $\mu^{*}$ is defined through

$$
\sum_{j} c_{j} x_{j}^{*}\left(\mu^{*}\right)-\bar{P}=0
$$

Equivalently, we need to construct a partition of the index set $\mathcal{J}$ as $\mathcal{J}=\mathcal{J}_{0} \cup \mathcal{J}_{p} \cup \mathcal{J}_{i}$ where $x_{j}^{*}$ attains the value 0 , $p_{j}$, or $G_{j}^{-1}\left(\mu^{*}\right)$ if it belongs to set $\mathcal{J}_{0}, \mathcal{J}_{p}$, or $\mathcal{J}_{i}$, respectively. In computational terms, viewing (7) as a function of $\mu$, this function is continuous and decreasing $\forall j$ so that the weighted sum of all such functions in (8) retains these properties since $c_{j} \geq 0$. Also, the left-hand side of (8), viewed as a function of $\mu$, changes signs in the interval $\left[\min _{j} G_{j}\left(p_{j}\right), \max _{j} G_{j}(0)\right]$. Hence, a simple bisection algorithm can be used for the numerical computation of $\mu^{*}$.

In principle, the analysis above solves $(\mathrm{P})$ in its most general case, and further insight may be gained by examining different dependence cases. Due to space constraints, the reader is referred to [12] for more details.

\section{NUMERICAL RESULTS}

\section{A. Independent Channels}

An extensive set of simulations was performed to test the relation between the various dependencies for the Shannon rate and BPSK BER utilities, for both variants (output and consumption). Due to space restrictions, only BER graphs for the output variant will be presented, with similar conclusions and observations holding for the Shannon rate and other variant. We assume $a$ and $b$ to be independent and exponentially distributed with an expected value ratio $r=\mathbb{E}\{a\} / \mathbb{E}\{b\}$ in the set $\{0.2,0.5,1,2,5\}$, respectively (different values are used to study the effect of channel asymmetry). The value of $g_{1}$ was set to 1 for all cases and the ratio $\bar{\gamma}_{1}=\mathbb{E}\{a\} / N_{1}$, which corresponds to the first-hop average SNR, was set to vary in the interval 0-30 dB. The exponential distribution allows for unbounded fading values; so, in order to keep the fading set bounded for simulation purposes, a 99.9\% confidence interval was used, which was then uniformly discretized into 500 states. This set contains all fading values of practical importance.

Setting $N_{1}, \eta$ to 1 , a set of eight simulations was performed, one for each $\left(\bar{P}_{2}, p_{2}\right)$ pair. The used pairs were $\{(0.01,0.1),(0.2,0.5),(0.2,2),(1,5),(1,10),(10,50)$, $(10,100),(100,500)\}$. The pairs were selected so that they cover a wide average power range (from very small to medium to large), and in certain cases, two pairs with the same average power but different peak power constraints were chosen so that the effect of the peak constraint could be determined. For each pair, the entire range $0-30 \mathrm{~dB}$ for $\bar{\gamma}_{1}$ was swept, and the optimal solutions for both dependencies were obtained. Besides the optimal solution, two other candidate solutions were evaluated. The first one was a modified inverse gain formula based on the first hop only and given by

$$
g_{2}^{*}(a, b)=g_{2}^{*}(a)=\frac{\min \left\{\bar{P}_{2}, p_{2}\right\}}{g_{1} a+N_{1}}
$$

for the output variant and

$$
g_{2}^{*}(a, b)=g_{2}^{*}(a)=\min \left(\frac{p_{2}}{g_{1} a+N_{1}}, \frac{\hat{P}_{2}}{g_{1} a+N_{1}}+1\right)
$$

for the consumption variant. The feasibility of both candidates is easy to verify (notice that in most practical cases, it holds $\left.\bar{P}_{2}, \hat{P}_{2}<p_{2}\right)$. The other candidate solution was obtained as follows: the problem of average SNR maximization was first solved for $a$ only dependence (i.e., we assumed $\Phi(x)=x$ ) resulting in a solution $g_{2 S}^{*}(a)$. This solution was then considered to be the solution to the original problem (with the true $\Phi$ utility). This solution was obviously suboptimal, but at the same time it allowed some algebraic manipulations, which slightly reduced the computational time. Additionally, since the average SNR is often used as a general (though not very descriptive) figure of merit for a receiver's performance [15], this solution can be regarded as a heuristic performance indicator. For notational purposes, we denote the solutions as " $a$ optimal" and " $(a, b)$ optimal" when we solve the original problem for each dependence type, and we use the terms "inverse" and "SNR" for the two (suboptimal) candidate solutions previously described.

The simulations resulted in a huge data set; thus, only the most representative cases will be given in graph form while the rest of the results will be verbally described. The following observations were made (unless otherwise stated, all facts hold for the entire 0-30-dB region) for the output variant.

- Channel asymmetry, as captured in the ratio $r$, affects system performance of a nonregenerative relay (since the relay always amplifies the noise of the first hop, it is important whether channel $a$ is significantly better or worse than channel $b$ ). The asymmetry becomes significant as $|r-1|$ increases (i.e., the performance difference between $r=0.5$ and $r=2$ is not as pronounced as when comparing $r=0.2$ and $r=5$ ). Hence, graphs will be presented only for the $r=0.2$ and $r=5$ ratios.

- For very large pairs, ${ }^{3}$ such as $(100,500)$ and to a lesser extent $(10,100)$, all solutions are extremely close to each other, which suggests that the profits of optimization are minimal in this case. This is not surprising, and can be seen as an example of the principle of diminishing returns when brute-force (i.e., high $\bar{P}_{2}$ ) is available.

- For all pairs, the "SNR" solution is less than an order of magnitude worse from the " $a$ optimal" one, which makes it an acceptable policy in practice.

- For all pairs, the inverse solution is extremely close to the " $a$ optimal" one, which suggests that if the relay observes $a$ only, the optimal solution is known in essentially closed form.

The last observation is somewhat unexpected and, considering its effect, extremely useful. It allows for a policy that achieves nearly optimal performance and can be dynamically applied in a trivial manner (since the relay needs to only observe the first hop) without any knowledge of the fading statistics. In order to assert whether this effect depends on the fading pdf,

\footnotetext{
${ }^{3}$ In terms of absolute power units, not necessarily their component ratio.
} 


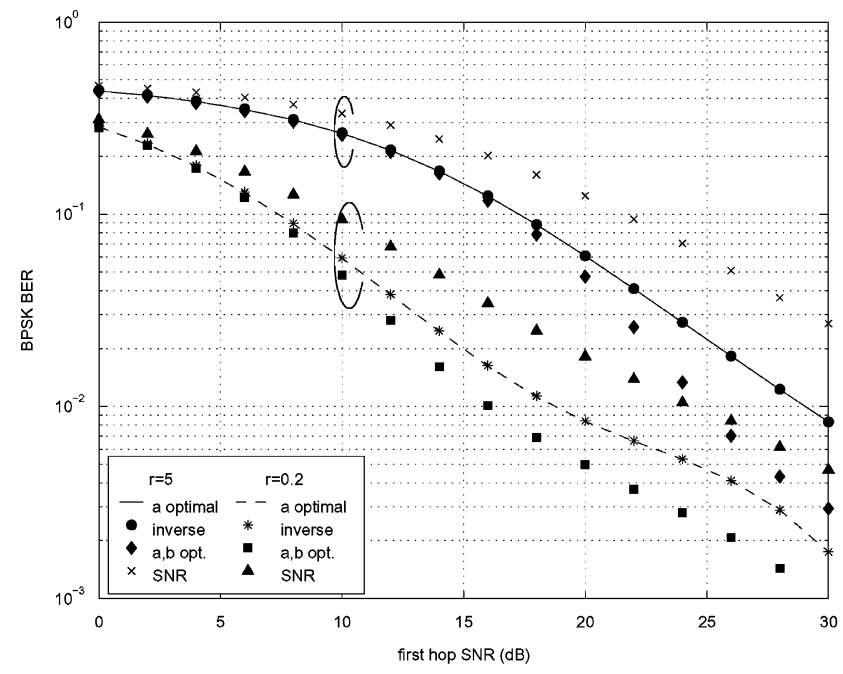

(a)

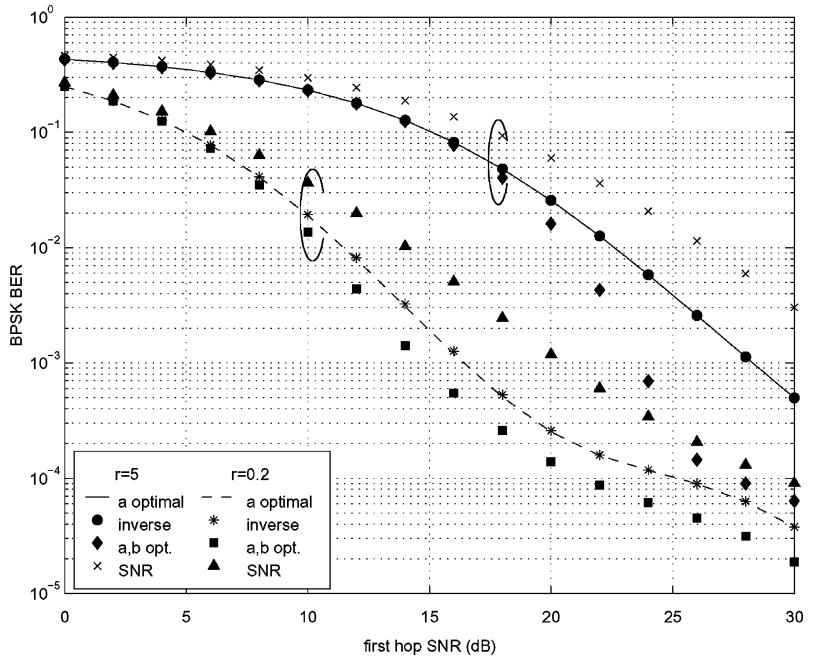

(b)

Fig. 1. Optimal BER versus inverse and SNR solutions for the pair $(0.2,2)$ and $r=0.2,5$. (a) Pair $(0.2,2)$-Exponential distribution. (b) Pair $(0.2,2)-$ Nakagami- $m$ distribution $(m=2)$.

simulations were performed for Nakagami- $m$ channels (with $m=0.5,2,4)$ using the same pairs and $r$ ratios, and similar results were obtained. A pair $(0.001,0.1)$ was also used and, for this case, there was an evident difference between the " $a$ optimal" and "inverse" solutions, although still within a factor of 2. The final conclusion, arising from the simulations only, was that for the $a$ only dependence, the inverse formula of (9) is practically the optimal solution provided that $\bar{P}_{2}$ is not too low.

This also makes a comparison between the " $a$ optimal" and " $(a, b)$ optimal" solutions imperative since, in case the performance difference is not decisively in favor of the latter, the "inverse" solution (which is very close to the " $a$ optimal" one) can be considered as a viable alternative due to its striking simplicity. Fig. 1 presents a comparison among all possible solutions for the pair $(0.2,2)$ (as previously mentioned, higher average power results in minimal performance gains while lower average power is rarely used in practice) both for exponential and Nakagami distributions. It is clear that the superiority margin of the " $(a, b)$ optimal" versus the " $a$ optimal" solution depends on the power constraints as well as the fading distribution. It is seen, however, that in all cases, the benefits of the " $(a, b)$ optimal" solution manifest themselves for relatively high first-hop SNR. An intuitive explanation is the following: It is clear from (1) that the SNR is a product of two terms, the latter of which (the one containing the $g_{2}$ contribution) is upper bounded by 1. Hence, if the first term of the SNR (the one containing the $a$ contribution) is too low, then any kind of optimization will not result in significant gains, since the maximum that can be achieved is already low in the first place.

Restricting our attention to the high SNR region (say $>20 \mathrm{~dB}$ ), it is evident that the performance increase from observing both $a, b$ is quite problem specific. For example, at 30 $\mathrm{dB}$, the " $(a, b)$ optimal" solution is better than the " $a$ optimal" by a factor of 10 for the pair $(0.2,2), r=0.2$ and Nakagami- $m$ fading with $m=2$, as shown in Fig. 1, while for the same pair and Nakagami- $m$ with $m=0.5$ (graph not shown), this factor is reduced to 2 . However, based on the performed simulations, it seems that significant gains from observing $a$ and $b$ are usually achieved for low, rather than high $r$ ratios. This suggests that observing both channels is essentially required when channel $b$ is generally much "worse" than channel $a$. The latter can be again explained by the same SNR-based intuitive argument as in the previous paragraph.

\section{B. Correlated Channels}

The case of dependent fading channels in relay systems has received little attention mostly due to the higher mathematical complexity it entails in its analytical treatment. However, in physical terms, a geographically homogeneous area, such as a flat valley or an urban area with buildings of similar type and height, would imply a dependence between the two channels (e.g., knowing that $a$ is high/low allows us to infer the range of b).

Although an analytical treatment is prohibitive, our framework is sufficiently general to handle this case numerically. Specifically, we consider the following scenario. Let $a$ and $b$ be dependent discrete RVs. If the conditional pdf $\pi_{b \mid a}$ is known a priori, problem (P) can be solved numerically with an optimal gain solution of $g_{\mathrm{dep}}$, where the appropriate dependence is assumed. If $\pi_{b \mid a}$ is not known, it must be estimated from direct channel measurements, but this would destroy the offline nature of our method, not to mention the additional power required for transmission of this information between nodes. Hence, we address the following question: What is the effect of falsely assuming dependent channels to be independent? In other words, if we always use the solution $g_{\text {in }}$ obtained for independent channels, what would the objective utility be compared to the $g_{\text {dep }}$ solution? To make this comparison on a fair basis, the marginal pdfs must obviously be the same in both cases, and the utility computed for $g_{\text {in }}$ must use the conditional $\pi_{b \mid a}$ pdf in the calculation. 
TABLE I

OPTIMAL BER FOR $a$ ONLY AND $a, b$ DEPENDENCE WHEN THE CHANNELS ARE FALSELY ASSUMED TO BE INDEPENDENT

\begin{tabular}{|c|c|c|c|c|}
\hline $\begin{array}{c}\text { first hop } \\
\text { SNR (dB) }\end{array}$ & $\rho=0.2$ & $\rho=0.4$ & $\rho=0.6$ & $\rho=0.8$ \\
\hline \multicolumn{5}{|c|}{ Optimal BER $\times 10(a$ only) } \\
\hline \multirow{2}{*}{0} & 3.1114 & 3.0708 & 3.0218 & 2.9606 \\
& 3.1299 & 3.0887 & 3.0402 & 2.9820 \\
\hline \multirow{2}{*}{8} & 1.1158 & 1.1038 & 1.0808 & 1.0395 \\
& 1.1629 & 1.1553 & 1.1410 & 1.1129 \\
\hline \multirow{2}{*}{16} & 0.1929 & 0.1904 & 0.1828 & 0.1652 \\
& 0.2671 & 0.2681 & 0.2688 & 0.2671 \\
\hline \multirow{2}{*}{24} & 0.0303 & 0.0297 & 0.0284 & 0.0257 \\
& 0.1267 & 0.1267 & 0.1265 & 0.1260 \\
\hline \multirow{5}{*}{0} & 3.0873 & 3.0474 & 3.0008 & 2.9454 \\
& 3.0919 & 3.0556 & 3.0108 & 2.9536 \\
\hline \multirow{2}{*}{4} & 2.0410 & 2.0140 & 1.9790 & 1.9307 \\
& 2.0503 & 2.0332 & 2.0082 & 1.9688 \\
\hline \multirow{2}{*}{8} & 1.0408 & 1.0344 & 1.0232 & 1.0019 \\
& 1.0474 & 1.0488 & 1.0474 & 1.0384 \\
\hline \multirow{2}{*}{12} & 0.3730 & 0.3772 & 0.3812 & 0.3836 \\
& 0.3738 & 0.3793 & 0.3859 & 0.3934 \\
\hline
\end{tabular}

For the case of $a$ only dependence, it is easy to see that the $g_{\text {in }}$ solution always satisfies the average power constraint of the dependent problem, since only $\pi_{a}$ appears in it. Hence, the utility obtained from $g_{\text {in }}$ will be suboptimal compared to $g_{\text {dep }}$. On the other hand, for $a, b$ dependence, the average power constraint involves $\pi_{a b}$, which is different between the dependent and independent cases $\left(\pi_{b \mid a} \pi_{a}\right.$ and $\pi_{a} \pi_{b}$, respectively). Therefore, for this dependence, a comparison between the utilities for $g_{\text {in }}$ and $g_{\text {dep }}$ can have two outcomes: either $g_{\text {in }}$ violates ${ }^{4}$ the average power constraint of the dependent case, or it satisfies the constraint, which by construction requires it to be suboptimal compared to $g_{\mathrm{dep}}$. The former physically means that the relay's average lifetime will be less than required while the latter is self-explanatory.

To address the previous questions, the case of joint exponential fading channels was considered [15, p. 142, eq. (6.2)]. The correlation coefficient $\rho$ was set to $0.2,0.4,0.6,0.8$ and the algorithm was applied to pair $(0.6,6)$ for the output variant. The findings are summarized in Table I, where the following convention is used. In each row, the upper/lower number represents the $g_{\text {dep }} / g_{\text {in }}$ utility, respectively, for the corresponding $\rho$. An inspection of the table reveals the following.

- For the $a$ only dependence, $g_{\text {in }}$ is always suboptimal as previously explained. For the BER utility, the difference between the two solutions increases with respect to $\bar{\gamma}_{1}$ and becomes significant for large $\bar{\gamma}_{1}$ (for example, at $24 \mathrm{~dB}$, the dependent BER is four times less than the independent BER for all $\rho$ ). Hence, assuming independent channels in these cases results in inferior performance to what could be potentially achieved.

- For the $a, b$ dependence, the average power constraint violation occurs for $\bar{\gamma}_{1} \geq 14 \mathrm{~dB}$, while for lower $\bar{\gamma}_{1}$, the

\footnotetext{
${ }^{4}$ Since the obtained solution is numerical, violates in this sense means that the average power is larger than $1+\delta$ times the constraint where $\delta$ is the iteration stopping criterion.
}

solution obtained by assuming independent channels is very close to the optimal $g_{\text {dep }}$.

From the previous discussion, the following dilemma arises regarding the relay's ability to adjust its gain based on either $a$ or both $a$ and $b$. Specifically, for sufficiently high $\bar{\gamma}_{1}$, if we monitor both channels, i.e., we compute $g_{2}(a, b)$, we must explicitly take dependence into account to satisfy the average power constraint, which in turn requires additional processing and transmissions. To avoid this, we can compute $g_{2}(a)$ assuming independent channels and get a utility very close to the truly optimal $g_{\text {dep }}(a)$ utility. The problem is that for high $\bar{\gamma}_{1}$, the $g_{2}(a)$ utility is usually much less than the $g_{2}(a, b)$ utility. Hence, in order to maximize performance in high $\bar{\gamma}_{1}$, we need to explicitly take dependence into account. The proposed method is general enough to allow for such cases.

\section{CONCLUSION}

This paper presented a general methodology for computing the utility-optimal CSI-based relay gain of a dual-hop system, subject to both average and instantaneous power constraints, for arbitrary fading pdfs. The emphasis was placed on examining the different dependencies of the gain on the channel states and their effect on system performance, as well as the practical importance of performing the optimization in the first place. Simulations were presented for the BER utility, which indicated that a modified "inverse gain" solution is very close to optimal except for certain cases that were demonstrated (and showed that a considerable performance gain is possible by exploiting both channel states in sufficiently high first-hop SNRs). The almost-optimality of the "inverse gain" solution was verified for many fading distributions, and was seen to be generally applicable with high confidence. This provides a good rule of thumb for optimal nonregenerative relay operation.

Furthermore, the effect on the system performance of assuming dependent channels to be independent was numerically investigated, and it was seen that this action is allowable for low first-hop SNR, in the sense that it leads to almost optimal utilities. However, as the first-hop SNR increases, it gradually becomes necessary to take the dependence into account if truly optimal solutions are desired. Future research includes treatment of the outage probability as a performance metric and the determination of the corresponding optimal relay gain in both regenerative and nonregenerative systems.

\section{REFERENCES}

[1] J. Laneman, D. Tse, and G. Wornell, "Cooperative diversity in wireless networks: Efficient protocols and outage behavior," IEEE Trans. Inf Theory, vol. 50, no. 12, pp. 3062-3080, Dec. 2004.

[2] V. Emamian, P. Anghel, and M. Kaveh, "Multi-user spatial diversity in a shadow-fading environment," in Proc. Veh. Technol. Conf., Sep. 2002, vol. 1, pp. 573-576.

[3] A. Sendonaris, E. Erkip, and B. Aazhang, "User cooperation diversityPart I: System description," IEEE Trans. Commun., vol. 51, no. 11, pp. 1927-1938, Nov. 2003 
[4] A. Sendonaris, E. Erkip, and B. Aazhang, "User cooperation diversitypart II: Implementation aspects and performance analysis," IEEE Trans. Commun., vol. 51, no. 11, pp. 1939-1948, Nov. 2003.

[5] M. Hasna and M. Alouini, "A performance study of dual-hop transmissions with fixed gain relays," IEEE Trans. Wireless Commun., vol. 3, no. 6, pp. 1963-1968, Nov. 2004.

[6] M. Hasna and M. Alouini, "Optimal power allocation for relayed transmissions over Rayleigh-fading channels," IEEE Trans. Wireless Commun., vol. 3, no. 6, pp. 1999-2004, Nov. 2004.

[7] G. Karagiannidis, "Performance bounds of multihop wireless communications with blind relays over generalized fading channels," IEEE Trans. Wireless Commun., vol. 5, no. 3, pp. 498-503, Mar. 2006.

[8] J. Boyer, D. Falconer, and H. Yanikomeroglu, "Multihop diversity in wireless relaying channels," IEEE Trans. Commun., vol. 52, no. 10, pp. $1820-1829$, Oct. 2004.

[9] J. Laneman and W. Wornell, "Distributed space-time-coded protocols for exploiting cooperative diversity in wireless networks," IEEE Trans. Inf. Theory, vol. 49, no. 10, pp. 2415-2425, Oct. 2003.
[10] G. Kramer, M. Gastpar, and P. Gupta, "Cooperative strategies and capacity theorems for relay networks," IEEE Trans. Inf. Theory, vol. 51, no. 9 , pp. 3037-3063, Sep. 2005.

[11] J. Laneman and G. Wornell, "Energy-efficient antenna sharing and relaying for wireless networks," in Proc. IEEE Wireless Commun. Netw., Sep. 2000, pp. 7-12.

[12] M. Gatzianas, L. Georgiadis, and G. Karagiannidis "Optimally allocated power regions of a dual-hop relay system for different CSI dependencies" (2006). [Online]. Available: http://users.auth.gr/ mgkatzia/regions. pdf.

[13] R. Berry and R. Gallager, "Communication over fading channels with delay constraints," IEEE Trans. Inf. Theory, vol. 48, no. 5, pp. 11351149, May 2002.

[14] D. Bertsekas, Nonlinear Programming. Belmont, MA: Athena Scientific, 1995.

[15] M. Simon and M. Alouini, Digital Communication Over Fading Channels: A Unified Approach to Performance Analysis. Hoboken, NJ: WileyInterscience, 2000 